ESAIM: PROCEEDINGS AND SURVEYS, September 2017, Vol. 59, p. 43-55

B. Bouchard, E. Gobet and B. Jourdain, Editors

\title{
OPTIMAL CONNECTIVITY FOR A LARGE FINANCIAL NETWORK ${ }^{*, * *, * * *}$
}

\author{
Rui Chen ${ }^{1}$, Andreea Minca ${ }^{2}$ And Agnès Sulem ${ }^{3}$
}

\begin{abstract}
We investigate network formation for a set of financial institutions represented as nodes. Linkages are source of income, and at the same time they bear the risk of contagion. The optimal connectivity of the nodes results from a game, in which the risk of contagion depends on the choices of all nodes in the system. Our financial network model can be interpreted as a set of banks connected through funding relations, in which a node's threshold to contagion is represented by its external funding capacity. A second interpretation is that of a set of insurers connected through reinsurance contracts, in which the threshold to contagion is represented by their capital. Our results show that when the threshold distribution across the nodes has higher variance, then, in equilibrium, the average connectivity is in general increasing, but the link failure probability decreases. This suggests that financial stability is best described in terms of the mechanism of network formation than in terms of simple statistics of the network topology like the average connectivity.
\end{abstract}

Résumé. On étudie la formation d'un réseau financier dont les noeuds représentent des institutions financières. Les liens sont sources de revenus mais également de risque de contagion de défaut. La connectivité optimale du réseau résulte d'un jeu, dans lequel le risque de contagion dépend de tous les noeuds du système. Notre réseau peut être interprété comme un ensemble de banques connectées entre elles par des relations de financement, le seuil de contagion de défaut pour une banque représentant ici sa capacité de financement extérieur. On peut aussi appliquer notre modèle à l'étude d'un réseau d'assureurs connectés par des contrats de réassurance, pour lesquels le seuil de contagion de chaque noeud est son capital. Nos résultats montrent que lorsque la distribution du seuil de contagion de défaut sur l'ensemble des noeuds a une grande variance, la connectivité moyenne croit, alors que la probabilité de défaut décroit. Cela suggère que l'étude de la stabilité financière d'un réseau financier doit prendre en compte le mécanisme de formation du réseau et pas seulement sa connectivité moyenne.

\section{INTRODUCTION}

Interconnected systems are subject to contagion in time of distress. Recent effort has been dedicated to understanding the relation between network topology and the scope of distress propagation, see e.g., [1, 2, 5, 16. and the references therein. It is critical to recognize that connectivity is a result of an optimization problem of agents, who derive benefits from connections and view the associated contagion risk as a cost. In the context

* This work was partially founded by a research grant from the Europlace Institute of Finance.

** Andreea Minca is partially funded under NSF grant CMMI 1638230.

*** Rui Chen is funded by a grant from Fondation Sciences Mathématiques de Paris.

1 INRIA Paris, 3 rue Simone Iff, CS 42112, 75589 Paris Cedex 12, France, and Université Paris-Dauphine, email: rui.a.chen@inria.fr

${ }^{2}$ School of Operations Research and Information Engineering, Cornell University, Ithaca, NY 14850, USA, email: acm299@cornell.edu

3 INRIA Paris, 3 rue Simone Iff, CS 42112, 75589 Paris Cedex 12, France, and Université Paris-Est, email: agnes.sulem@inria.fr

(c) EDP Sciences, SMAI 2017 
of financial systems, this benefit-cost view of connections is first presented in [5], who analyze socially optimal network topologies.

A threshold model of contagion for heterogenous graphs is proposed in 11. Each node represents a bank, insurance company or institution, and is endowed with a threshold to contagion. A set of nodes fail exogenously and any node in the system can fail due to contagion when its threshold is surpassed by the number of connected nodes that fail. The availability of asymptotic results on the fraction of nodes that fail at the end of the contagion process allows us to estimate the failure probability for a network with given connectivity. In this paper, we take a step further and find the equilibrium choice of connectivity. The banks' payoff is impacted by their own choice of connectivity, which governs both the benefits from linkages as well as the exposure to the risk of contagion in the network. The risk of contagion in turn depends on all the banks' connectivity choices.

The fundamentals in the model are represented by the thresholds. Under full information, the distribution of the thresholds across banks is common knowledge. Once a node chooses its connectivity, the network is generated using the configuration model. This is a random graph chosen uniformly over all graphs with the given connectivity sequence across nodes. For this random graph, a law of large numbers gives the limit probability that a randomly chosen link fails, i.e. it links to a failed node. This probability depends on the join distribution of the connectivity and thresholds. Our equilibrium concept is a rational expectations equilibrium. For an anticipated link failure probability, nodes choose their optimal connectivity as a function of their threshold. We then ensure that the anticipated link failure probability is equal to the failure probability in the network with the chosen connectivity.

Our equilibrium model is quite different from past literature. In [5] the authors use the notion of pairwise Nash stability and a contagion mechanism in which nodes fail with an exogenous probability when a neighbor fails. The notion of pairwise Nash stability is also used in [8. Here we exploit the asymptotic results for contagion scope in our particular choice of random graph, where connectivity is optimized but the actual neighbors are randomly chosen. We are interested in the optimal connectivity as a function of fundamentals, namely the thresholds. Our notion of equilibrium can be seen as a Nash equilibrium with a continuum of players, classified according to their thresholds and we obtain a unique such equilibrium.

For the asymptotic case, our results allow us to understand how the degree of heterogeneity in the fundamentals translates into failure risk in the network with endogenous connectivity. Nodes adjust their connectivity to the degree of heterogeneity in the fundamentals. When the fundamentals are more homogenous, then the average connectivity is lower, but the failure probability is higher than in the case when there is more dispersion in the fundamentals. The network payoff is defined as the average number of links times the survival probability. For different fundamentals, the network connectivity is adjusted in equilibrium to yield the same network payoffs. However, these networks are not equivalent from the point of view of systemic risk. To obtain lower default probability and systemic risk, more dispersion in fundamentals is preferable. In this case, nodes with large thresholds would act as stabilizers in time of distress. Our results are in line with 8 , who obtain in a different model that economies that are fundamentally "safer", in the sense that they are subject to less volatile shocks, generate higher interconnectedness. Here, it is the homogeneity of the thresholds, or otherwise said their lesser variance, that generates more connectivity.

Our paper is complementary to the line of research on the control of contagion, e.g., [3, 14, 15. In these works, a central party, for example a regulator or government, seeks to minimize contagion. In [7 the author explores the effect of moral hazard on network topology, as the network is formed with the anticipation of government bailouts in case of distress. Parallel to the development of network models for systemic risk, a recent series of works [6, 9 11, 11] introduced a reduced form approach to systemic risk analysis, based on mean field interaction models. In these works, the trajectories of the banks are modeled as a set of coupled diffusions, which may be controlled by a central party that specifies the parameters of the interaction. Here in contrast, the financial institutions themselves are the decision makers, and their decision is made before the shock, with a rational expectation on the way the cascade will evolve following the shock.

While most of the work on contagion in financial networks refers to banking networks, a recent literature 4, 13 , considers insurance-reinsurance networks. Contagion proceeds similarly in such networks, as failed reinsurers 
cannot honor contracts to other institutions, and such failures can propagate via chains of reinsurance contracts. Our work offers thus guidance on the formation of such networks and their inherent risk.

The paper is organized as follows. In section 1 we present the model of contagion and the nodes' optimization criteria. In section 2 we investigate the existence and uniqueness of the optimal connectivity, under an anticipated link failure probability. In Section 3 we analyze the equilibrium connectivity. Finally, in Section 4 we study numerically the equilibrium choice as a function of fundamentals.

\section{Node Performance under Linkage Benefits AND CONTAGion RISK}

We represent a financial system by $n$ institutions (nodes), endowed with a sequence of thresholds $(\theta(i))_{i \in[n]}$. We can interpret the nodes as banks, in which case the thresholds represent the funding that these banks can raise from external lenders. Banks loan to other banks, and thus choose their connectivity and form funding linkages. Alternatively, we can interpret the nodes as insurance companies. Their threshold represents their capital. They can reinsure other insurance companies, and to do so they choose their connectivity and form reinsurance linkages.

Given a connectivity $\lambda(i), i \in[n]$, nodes form links according to the random matching from the configuration model. In the configuration model with given degree sequence $\lambda(i), i \in[n]$, each node $i$ is assigned $\lambda(i)$ incoming half edges and $\lambda(i)$ outgoing half edges. The (multi)graph results from uniform matching of the in-coming half edges and the outgoing half edges. As $n \rightarrow \infty$, self loops and parallel edges become rare, and the graph is simple with positive probability [12. This means that any property that holds with high probability on the configuration model, also holds with high probability conditional on this random graph being simple. This graph is denoted by $\mathcal{G}$ and we write $(i, j) \in \mathcal{G}$ for the event that there's a link between $i$ and $j$. We let $\mu_{n}(\lambda, \theta)$ be the fraction of nodes with degree $\lambda$ and threshold $\theta$,

$$
\mu_{n}(\lambda, \theta):=\frac{\{i \in[n] \mid \theta(i)=\theta, \lambda(i)=\lambda\}}{n} .
$$

We assume the following regularity conditions $\mu_{n}(\lambda, \theta) \rightarrow \mu(\lambda, \theta)$, as $n$ tends to $\infty$. We let the fraction of nodes with threshold $\theta$, be defined as

$$
\mu(\theta):=\sum_{\lambda} \mu(\lambda, \theta)
$$

This network is subject to contagion risk. After the network is formed, a shock occurs and a set $\mathcal{D}_{0}$, representing a fraction $\epsilon$ of the entire system, switches its threshold to zero. If the financial network represents a network of funding relations among banks, the initial shock has the meaning that the debt capacity of certain banks becomes zero, i.e., no party lends to them, so they exogenously become illiquid. If the financial network represents a network of reinsurance contracts among insurance companies, then the initial shock is to be interpreted that the capital of certain insurers becomes zero following an extreme event. Under both of these representations, the thresholds becoming zero is equivalent to the failure of the node.

This initial set of failures triggers a cascade of failures, as we assume that failed nodes cut linkages from the nodes connected to them. Banks that fail after becoming illiquid cut loans from other banks in the system. These banks in turn also fail if their debt capacity is smaller than the amount of funding that was cut by failed banks. Insurers that fail after their capital becomes zero cut the reinsurance of other insurers. These other insurers in turn also fail if their capital is smaller than the amount of reinsurance that was cut by failed insurers. In sum, whenever a the node's threshold is smaller than the number of linkages that are cut from it, then it fails due to contagion.

We have $\mathcal{D}_{1}:=\left\{j \in \mathcal{D}_{0}^{c} \mid \theta(j)<\sum_{i \in \mathcal{D}_{0}} \mathbb{1}_{(i, j) \in \mathcal{G}}\right\}$, with ${ }^{c}$ denoting set complement. The cascade progresses according to $\mathcal{D}_{n}:=\left\{j \in \mathcal{D}_{n-1}^{c} \mid \theta(j)<\sum_{i \in \mathcal{D}_{n-1}} \mathbb{1}_{(i, j) \in \mathcal{G}}\right\}$, and it stops after at most $n$ steps. We let $\mathcal{D}_{f}$ be the set of failures at the end of contagion process. We have the following theorem, which characterizes the set of failures. 
Theorem 1 ([1]). Let $p^{*}$ be the smallest fixed point of the map $J$ in [0,1], where

$$
J(p):=\sum_{\theta, \lambda} \frac{\lambda \mu(\theta, \lambda)}{\sum_{\theta, \lambda} \lambda \mu(\theta, \lambda)} \cdot B(\theta, \lambda, p)
$$

with

$$
B(\theta, \lambda, p):=\mathbb{P}(\operatorname{Bin}(\lambda, p) \geq \theta)=\sum_{l \geq \theta}^{\lambda}\left(\begin{array}{l}
\lambda \\
l
\end{array}\right) p^{l}(1-p)^{\lambda-l}
$$

Here Bin denotes a random variable with binomial distribution with parameters $\lambda$ and $p$.

(i) If $p^{*}=1$, i.e., if $J(p)>p$ for all $p \in[0,1)$, then asymptotically (when $n \rightarrow \infty$ ) almost all nodes fail during the cascade.

(ii) If $p^{*}<1$ and $p^{*}$ is a stable fixed point of $J$, i.e., $J^{\prime}\left(p^{*}\right)<1$, then the final fraction of failures $\frac{\left|\mathcal{D}_{f}\right|}{n}$ converges in probability to $\sum_{\lambda, \theta} \mu(\lambda, \theta) \sum_{\theta=0}^{j} B\left(\theta, \lambda, p^{*}\right)$.

The asymptotic fraction of surviving nodes with degree $\lambda$ and threshold $\theta$ at the end of the contagion is given (in the limit) by

$$
s^{\theta, \lambda}=\mu(\theta, \lambda)\left(1-B\left(\theta, \lambda, p^{*}\right)\right) .
$$

Remark 2. Consider the fixed point equation $p^{*}=J\left(p^{*}\right)$ and multiply both sides with $n \sum_{\theta, \lambda} \lambda \mu(\theta, \lambda)$, which represents the total number of links in the network. Then $p^{*} n \sum_{\theta, \lambda} \lambda \mu(\theta, \lambda)$ represents the number of failed links (links from failed nodes) present in the system. This must be equal at the end of the cascade to $\sum_{\theta, \lambda} \lambda n \mu(\theta, \lambda) B\left(\theta, \lambda, p^{*}\right)$, which gives the sum across nodes with different $\theta$ of their failed links. In other words, all failed links have been "accounted for" in terms of the nodes which are the counterparties.

This result is established by describing the contagion process using a Markov chain of lower dimension than the initial system, in which we aggregate nodes according to their connectivity, threshold, and number of failed counterparties. From the point of view of the evolution of the cascade, the nodes in the same class are indistinguishable. We then show that, as the network size increases, the Markov chain rescaled by network size converges in probability to a limit described by a system of ordinary differential equations, which can be solved in closed form. This readily gives us the asymptotic fraction of surviving nodes (in each class of connectivity and threshold) at the end of the cascade, namely $s^{\theta, j}$.

It is clear that any node in the network is exposed to global failure risk captured by $p^{*}$. We refer to $p^{*}$ as the global failure probability of a link. The failure probability of a node with degree $\lambda$ and threshold $\theta$ is $B\left(\theta, \lambda, p^{*}\right)$. As nodes add more connectivity, they increase the risk of contagion. Of course, nodes add connectivity because they derive benefits from their linkages. We capture these benefits in a simple way, by assuming that there exists a numéraire and that surviving nodes with connectivity $\lambda$ receive $\lambda$ (units of the numéraire) at the end of the cascade. They receive no benefit from their linkages if they fail.

Remark 3. Note that the ability to define a global failure probability depends on our choice of the configuration model as an underlying network. The configuration model is a flexible setting as it gives the possibility to use any degree distribution, but it carries the implicit assumption that a linkage default probability is independent of the end node in equilibrium. It remains an open problem to extend the equilibrium analysis below to other types of random graphs, e.g. inhomogeneous random graphs, and for case when the global failure probability is replaced by a failure probability matrix (with the default probability of a link depending on the types of its end nodes).

We are now ready to define the nodes' performance measure. 
Definition 4 (Nodes' performance). We define the performance criterion for a node with degree $j$ and threshold $\theta$ as the expected benefit of linkages, and it is given in the asymptotic limit by

$$
\lambda\left(1-B\left(\theta, \lambda, p^{*}\right)\right)
$$

with $p^{*}$ given in Theorem 1 .

Note that the global failure probability of a link $p^{*}$ depends on the connectivity choice of all nodes. Therefore, the optimal connectivity is an outcome of an equilibrium. We proceed in two steps to determine this equilibrium. In the first step, we let the nodes choose their connectivity according to an expected failure probability $p$ of a link, i.e., a node with threshold $\theta$ chooses a connectivity

$$
\lambda^{*}(\theta, p) \in \arg \max _{\lambda} \lambda(1-B(\theta, \lambda, p))
$$

In the second step, detailed in Section 3, we will impose an equilibrium condition that the expected failure probability of a link coincides with the actual failure probability of a link, under the optimal connectivity.

Before we proceed, we note that the banks maximize an expected payoff which is equal to the connectivity if the bank doesn't fail and 0 if it does. One could argue that in the performance criterion banks should only count the profit from the surviving linkages. In fact, whether we should use the expected benefit from all linkages or the expected benefit from the surviving links depends on the model interpretation. It can be of interest to use the expected benefit and not the expected benefit from the surviving links when surviving banks replace the defaulted links with new funding from outside creditors to maintain their business. In such case the replaced linkages have the same function as the original ones.

One could also assume that failed links do not provide any benefit and maximize the product of the probability of survival $1-B(\theta, \lambda, p)$ and the expectation of non-failed links $\lambda(1-p)$. The next remark shows that for fixed failure probability $p$ of a link, the banks' choice remains the same. This in turn implies the equilibrium and its stability properties remain the same.

Remark 5 (Robustness to the performance criterion). We can also define our performance criterion as the expected benefit from the survived links. There are two possible criteria. For fixed $\theta$ and $p$,

- $V_{1}(\lambda):=(1-p) \lambda(1-B(\theta, \lambda, p))$. In this case the optimizer remains the same as above since

$$
\max _{\lambda} V_{1}(\lambda)=(1-p) \max _{\lambda} \lambda(1-B(\theta, \lambda, p))
$$

Thus

$$
\arg \max _{\lambda} V_{1}(\lambda)=\arg \max _{\lambda} \lambda(1-B(\theta, \lambda, p)) .
$$

- $V_{2}(\lambda):=\sum_{l<\theta}(\lambda-l)\left(\begin{array}{l}\lambda \\ l\end{array}\right) p^{l}(1-p)^{\lambda-l}$. Straightforward computation shows that

$$
V_{2}(\lambda)=\sum_{l<\theta} \lambda\left(\begin{array}{c}
\lambda-1 \\
l
\end{array}\right) p^{l}(1-p)^{\lambda-l}=(1-p) \lambda(1-B(\theta, \lambda-1, p))
$$

Thus

$$
\arg \max _{\lambda} V_{2}(\lambda)=\arg \max _{\lambda} \lambda(1-B(\theta, \lambda-1, p))
$$

which shares the same structure as the original criterion and will lead to similar results.

Which criterion is more suitable depends on the contagion model. When the direction of link is the same as the direction of contagion (i.e. $i$ loans to $j$ and there is contagion from $i$ to $j$ and the link $(i, j)$ fails when $i$ withdraws funding from $j$ ) then $V_{1}$ represents the expected benefit of the non-failed links of the surviving banks. 
When the direction of the link is inverse to the direction of contagion (i.e. $i$ loans to $j$ and there is contagion from $j$ to $i$ and the link $(i, j)$ fails because $j$ is insolvent or cannot pay the loan) then $V_{2}$ represents the expected benefit of the non-failed links of the surviving banks.

\section{Nodes' optimal CONNECTIVity CHOICE}

In this section we investigate existence and uniqueness of the node's optimal connectivity. We begin by noting that the node's optimization problem carries a tradeoff. We recognize here that $1-B(\theta, \lambda, p)$ is equal to the Incomplete beta function $I_{1-p}(\lambda-\theta+1, \theta)$. We recall that $I_{x}(a+1, b)=I_{x}(a, b)-\frac{x^{a}(1-x)^{b}}{a \mathcal{B}(a, b)}$, where $\mathcal{B}(a, b)=\int_{0}^{1} x^{a-1}(1-x)^{b-1} d x$. We have $I_{1-p}(\lambda-\theta+2, \theta)=I_{1-p}(\lambda-\theta+1, \theta)-\frac{(1-p)^{\lambda-\theta+1} p^{\theta}}{(\lambda-\theta) \mathcal{B}(\lambda-\theta+1, \theta)}$ and thus $1-B(\theta, \lambda+1, p)<1-B(\theta, \lambda, p)$, which means that the survival probability decreases in $\lambda$.

The node's performance as a function of connectivity can now be written as

$$
V(\lambda):=\lambda \cdot I_{1-p}(\lambda-\theta+1, \theta)
$$

It is easy to see that this performance balances more benefits from connectivity (via the factor $\lambda$ ) against more risk, via the decreasing surviving probability $I_{1-p}(\lambda-\theta+1, \theta)$.

Proposition 6 (Existence). The optimization problem admits a finite optimizer $\lambda^{*}(\theta, p)$ which lies in the interval $\left[\frac{1-p}{p} \vee \theta, \frac{\theta-2 p+\sqrt{(\theta-2 p)^{2}+4(1-p) p}}{2 p}\right]$.

Proof. We will show that the node's performance $V(\lambda)$ is decreasing in $\lambda$ for $\lambda>\frac{\theta-2 p+\sqrt{(\theta-2 p)^{2}+4(1-p) p}}{2 p}$ and is increasing for $\lambda<\frac{1-p}{p} \vee \theta$. Recall that $\left(\begin{array}{c}\lambda+1 \\ l\end{array}\right)=\frac{\lambda+1}{\lambda+1-l}\left(\begin{array}{l}\lambda \\ l\end{array}\right)=\frac{1}{1-l /(\lambda+1)}\left(\begin{array}{l}\lambda \\ l\end{array}\right)$. We have

$$
V(\lambda+1)-V(\lambda)=\sum_{l \leq \theta-1}\left[(\lambda+1) \frac{1-p}{1-l /(\lambda+1)}-\lambda\right]\left(\begin{array}{c}
\lambda \\
l
\end{array}\right)(1-p)^{\lambda-l} p^{l} .
$$

Now if $\lambda>\frac{-(2-\theta)+2(1-p)+\sqrt{(2-\theta-2+2 p)^{2}+4(1-p) p}}{2 p}=\frac{\theta-2 p+\sqrt{(\theta-2 p)^{2}+4(1-p) p}}{2 p}$, then we have $(\lambda+1) \frac{1-p}{1-(\theta-1) /(\lambda+1)}-$ $\lambda<0$. This gives $(\lambda+1) \frac{1-p}{1-l /(\lambda+1)}-\lambda<0$, for all $l \leq \theta-1$ since $(\lambda+1) \frac{1-p}{1-l /(\lambda+1)}-\lambda$ is increasing in $l$. This leads to $(\lambda+1)^{2}(1-p)<\lambda(\lambda+2-\theta)$. Thus $\lambda>\frac{-(2-\theta)+2(1-p)+\sqrt{(2-\theta-2+2 p)^{2}+4(1-p) p}}{2 p}=\frac{\theta-2 p+\sqrt{(\theta-2 p)^{2}+4(1-p) p}}{2 p}$. Therefore if $\lambda>\frac{\theta-2 p+\sqrt{(\theta-2 p)^{2}+4(1-p) p}}{2 p}$, then $V(\lambda+1)-V(\lambda)<0$. Thus the optimal $\lambda^{*}$ should lie in the interval $\left[0, \frac{\theta-2 p+\sqrt{(\theta-2 p)^{2}+4(1-p) p}}{2 p}\right]$.

Similarly if $\lambda<\frac{1-p}{p}$, then $(\lambda+1)(1-p)-\lambda>0$ and thus we have $(\lambda+1) \frac{1-p}{1-l /(\lambda+1)}-\lambda>0$ for all $l \leq \theta-1$, again by the monotonicity in $l$. This gives that $V(\lambda+1)-V(\lambda)>0$ which means the optimal $\lambda^{*}$ should be greater than $\frac{1-p}{p}$. Also it is obvious to choose $\lambda^{*} \geq \theta$ for players with threshold $\theta$. We conclude that the optimal $\lambda^{*}$ should lie in the interval $\left[\frac{1-p}{p} \vee \theta, \frac{\theta-2 p+\sqrt{(\theta-2 p)^{2}+4(1-p) p}}{2 p}\right]$.

Corollary 7. The optimal connectivity lies in $\left[\frac{1-p}{p} \vee \theta, \frac{\theta}{p}-1\right]$.

Proof. This follows from Proposition 6 and the fact that $\frac{\theta-2 p+\sqrt{(\theta-2 p)^{2}+4(1-p) p}}{2 p}<\frac{\theta}{p}-1$. 
Remark 8. The same results hold for the performance criterion $V_{2}$. We mirror the proof of the existence result, Proposition 6. We have

$$
V_{2}(\lambda+1)-V_{2}(\lambda)=\sum_{l \leq \theta-1}\left[(\lambda+1) \frac{1-p}{1-l / \lambda}-\lambda\right]\left(\begin{array}{c}
\lambda-1 \\
l
\end{array}\right)(1-p)^{\lambda-1-l} p^{l}
$$

If $\lambda>\frac{\theta-p}{p}$, then we have $(\lambda+1) \frac{1-p}{1-(\theta-1) / \lambda}-\lambda<0$. This gives $(\lambda+1) \frac{1-p}{1-l / \lambda}-\lambda<0$, for all $l \leq \theta-1$ since $(\lambda+1) \frac{1-p}{1-l / \lambda}-\lambda$ is increasing in l. This leads to $(\lambda+1)(1-p)<\lambda+1-\theta$. Thus $\lambda>\frac{\theta-p}{p}$. Therefore if $\lambda>\frac{\theta-p}{p}$, then $V_{2}(\lambda+1)-V_{2}(\lambda)<0$. Thus the optimal $\lambda^{*}$ should lie in the interval $\left[0, \frac{\theta}{p}-1\right]$.

Similarly if $\lambda<\frac{1-p}{p}$, then $(\lambda+1)(1-p)-\lambda>0$ and thus we have $(\lambda+1) \frac{1-p}{1-l / \lambda}-\lambda>0$ for all $l \leq \theta-1$, again by the monotonicity in $l$. This gives that $V_{2}(\lambda+1)-V_{2}(\lambda)>0$ which means the optimal $\lambda^{*}$ should be greater than $\frac{1-p}{p}$. Also it is obvious to choose $\lambda^{*} \geq \theta$ for players with threshold $\theta$. We conclude that the optimal $\lambda^{*}$ lies in the interval $\left[\frac{1-p}{p} \vee \theta, \frac{\theta}{p}-1\right]$.

\section{Equilibrium}

We now impose the equilibrium condition, that the failure probability given by Theorem 1 with nodes at their optimal connectivity coincides with the anticipated failure probability.

Definition 9. We call $\left(p^{*},\left(\lambda^{*}(\theta), \theta \geq 0\right)\right)$ an standard equilibrium if

- Given $p^{*}$,

$$
\lambda^{*}(\theta) \in \arg \max _{\lambda} \lambda\left(1-B\left(\theta, \lambda, p^{*}\right)\right), \text { for each } \theta
$$

- $p^{*}$ is the smallest solution of the fixed point equation :

$$
p^{*}=\sum_{\theta} \frac{\lambda^{*}(\theta) \mu(\theta)}{\sum_{\theta} \lambda^{*}(\theta) \mu(\theta)} \cdot B\left(\theta, \lambda^{*}(\theta), p^{*}\right) .
$$

The fixed point equation in the equilibrium definition is derived from the fixed point equation in Theorem 1. where the connectivity is set to $\lambda^{*}(\theta)$ and we note that $\mu(\theta)=\mu\left(\theta, \lambda^{*}(\theta)\right)$.

Combining the two conditions above, we see that in equilibrium the failure probability of a link $p^{*}$ is the smallest fixed point of the map

$$
\phi(p):=\sum_{\theta} \frac{\lambda^{*}(\theta, p) \mu(\theta)}{\sum_{\theta} \lambda^{*}(\theta, p) \mu(\theta)} \cdot B\left(\theta, \lambda^{*}(\theta, p), p\right) .
$$

Now we study the existence of the equilibrium.

Proposition 10 (Existence of equilibrium). When $\lambda^{*}(\theta, p)$ is continuous in $p \in[0,1)$, the function $\phi$ admits at least one fixed point $p^{*}$. When $\lambda^{*}(\theta, p)$ is not continuous in $p$, the map $\phi$ admits a "relaxed" fixed point $\hat{p}$ defined as $\hat{p}:=\min \{p, \phi(p) \leq p, p \in[0,1)\}$. For both cases, we let $\lambda^{*}(\theta)=\lambda^{*}\left(\theta, p^{*}\right)$.

Proof. The proof is immediate: when $\lambda^{*}(\theta, p)$ is continuous in $p \in[0,1)$ then the function $\phi$ is continuous in $[0,1)$. It thus admits at least one fixed point since $\lim _{p \rightarrow 1} \phi(p) \leq \sum_{\theta} \mu(\theta) \frac{\lambda^{*}(\theta, p)}{\sum_{\theta} \lambda^{*}(\theta, p) \mu(\theta)}=1$ and $\lim _{p \rightarrow 0} \phi(0) \geq 0$. When $\lambda^{*}(\theta, p)$ is not continuous, the relaxed fixed point always exists since $\phi(0) \geq 0$ and $\phi(1) \leq 1$.

Remark 11. The continuity of $\lambda^{*}(\theta, p)$ in $p$ is guaranteed by the uniqueness of the optimal connectivity $\lambda^{*}(\theta, p)$. However, this uniqueness is difficult to show for problem (1). We provide below a uniqueness result for an 
approximated problem obtained by approximating the binomial distribution in (1) by the Poisson distribution $\operatorname{Pois}(\lambda p)$ when $\lambda$ is large enough and $p$ is small :

$$
\lambda^{*}(\theta, p) \in \arg \max _{\lambda} \lambda(1-\operatorname{Pois}(\theta, \lambda, p)),
$$

where $1-\operatorname{Pois}(\theta, \lambda, p)=\sum_{k=0}^{\theta-1} \frac{(\lambda p)^{k} e^{-\lambda p}}{k !}$.

Proposition 12 (Uniqueness). The approximated optimization problem (5) admits an unique solution $\lambda^{*}$.

Proof. Recall that $\left(\frac{(\lambda p)^{k} e^{-\lambda p}}{k !}\right)^{\prime}=p\left(\frac{(\lambda p)^{k-1} e^{-\lambda p}}{(k-1) !}-\frac{(\lambda p)^{k} e^{-\lambda p}}{k !}\right)$. Thus

$$
\begin{aligned}
\frac{d[\lambda(1-\operatorname{Pois}(\theta, \lambda, p))]}{d \lambda}= & \sum_{k=0}^{\theta-1} \frac{(\lambda p)^{k} e^{-\lambda p}}{k !}-\lambda p e^{-\lambda p}+\lambda \sum_{k=1}^{\theta-1} p\left(\frac{(\lambda p)^{k-1} e^{-\lambda p}}{(k-1) !}-\frac{(\lambda p)^{k} e^{-\lambda p}}{k !}\right) \\
& =\sum_{k=0}^{\theta-1} \frac{(\lambda p)^{k} e^{-\lambda p}}{k !}-\lambda p \cdot \frac{(\lambda p)^{\theta-1} e^{-\lambda p}}{(\theta-1) !} .
\end{aligned}
$$

The optimal $\lambda^{*}$ should satisfy

$$
\sum_{k=0}^{\theta-1} \frac{(\lambda p)^{k}}{k !}=\frac{(\lambda p)^{\theta}}{(\theta-1) !}
$$

We note that if $\lambda \geq \frac{\theta+1}{p}$, then $\frac{(\lambda p)^{k+1}}{(k+1) !}>\frac{(\lambda p)^{k}}{k !}$, for $k=0,1, \ldots \theta-1$. Thus,

$$
\sum_{k=0}^{\theta-1} \frac{(\lambda p)^{k}}{k !}<\theta \frac{(\lambda p)^{\theta}}{\theta !}=\frac{(\lambda p)^{\theta}}{(\theta-1) !}
$$

So we must have $\lambda^{*}<\frac{\theta+1}{p}$. It follows that

$$
\frac{d^{2}[\lambda(1-\operatorname{Pois}(\theta, \lambda, p))]}{d \lambda^{2}}=-p \cdot \frac{(\lambda p)^{\theta-1} e^{-\lambda p}}{(\theta-1) !}-p(\theta) \frac{(\lambda p)^{\theta-1} e^{-\lambda p}}{(\theta-1) !}+p \frac{(\lambda p)^{\theta} e^{-\lambda p}}{(\theta-1) !}<0 .
$$

Thus $\lambda(1-\operatorname{Pois}(\theta, \lambda, p))$ is concave in $\lambda$ and uniqueness of the optimizer follows.

\section{Remark 13. Relation to the Nash equilibrium}

We can relate the equilibrium of Definition 9 to a Nash equilibrium of the following game. Any player with threshold $\theta^{0}$, given the connectivity $\{\lambda(\theta)\}_{\theta \neq \theta^{0}}$ of the other players, computes its optimal connectivity

$$
\lambda^{*}\left(\theta^{0}, p\right) \in \arg \max _{\lambda} \lambda\left(1-B\left(\theta^{0}, \lambda, p\right)\right)
$$

under the constraint that

$$
\sum_{\theta \neq \theta^{0}} \mu(\theta) \frac{\lambda(\theta)}{\sum_{\theta \neq \theta^{0}} \mu(\theta) \lambda(\theta)+\mu\left(\theta^{0}\right) \lambda^{*}\left(\theta^{0}\right)} B(\theta, \lambda(\theta), p)+\mu\left(\theta^{0}\right) \frac{\lambda^{*}\left(\theta^{0}\right)}{\sum_{\theta \neq \theta^{0}} \mu(\theta) \lambda(\theta)+\mu\left(\theta^{0}\right) \lambda^{*}\left(\theta^{0}\right)} B\left(\theta^{0}, j, p\right)=p .
$$

We can rewrite the above constraint $p=G\left(\lambda^{*}(\theta)_{\theta \neq \theta^{0}}, \lambda\right)$ for some function $G$. Thus, the optimization criterion can be rewritten as :

$$
\lambda^{*}(\theta) \in \arg \max _{\lambda} \lambda\left[1-B\left(\lambda, \theta^{0}, G\left(\lambda^{*}(\theta)_{\theta \neq \theta^{0}}, \lambda\right)\right)\right] .
$$

In this sense, $\left(\lambda^{*}(\theta), \theta \geq 0\right)$ is a Nash equilibrium. 


\subsection{Analysis of the equilibrium}

Case 1: The system is perfectly observable to all the nodes, i.e. all the $\theta$ and their distribution $\mu(\theta)$ are common knowledge. Then all the nodes will simultaneously choose their connectivity in the equilibrium state by rational expectations and the system simultaneously reaches the equilibrium $\left(\lambda^{*}(\theta), \theta \geq 0\right)$, by virtue of Proposition 10.

Case 2: The system is only partially observed by the players, for example each player only knows his own threshold $\theta$. All the players optimally choose their linkage $\lambda^{*}\left(\theta, p^{0}\right)$ according to some anticipated link failure probability $p^{0}$ (this could be the failure probability estimated from the current environment). Then the link failure probability $p^{1}$ in the next stage is determined by the fixed point of the map $p \rightarrow F\left(p^{0}, p\right)$, where

$$
F\left(p^{0}, p\right):=\sum_{\theta} \mu(\theta) \frac{\lambda^{*}\left(\theta, p^{0}\right)}{\sum_{\theta} \mu(\theta) \lambda^{*}\left(\theta, p^{0}\right)}\left[1-B\left(\theta, \lambda^{*}\left(\theta, p^{0}\right), p\right)\right] .
$$

Then all nodes adjust their expectation of link failure probability from $p^{0}$ to $p^{1}$ and choose connectivity $\lambda^{*}\left(\theta, p^{1}\right)$ corresponding to $p^{1}$. This will be further updated to a series of link failure probabilities $\left\{p^{0}, p^{1} \ldots p^{n}\right\}$.

We conjecture that the sequence $\left\{p^{0}, p^{1} \ldots p^{n}\right\}$ will not convergence to the equilibrium states $p^{*}$, since the following map $H$ is divergent near the equilibrium state

$$
H: p^{0} \rightarrow p^{1}=\min \left\{p \mid \sum_{\theta} \mu(\theta) \frac{\lambda^{*}\left(\theta, p^{0}\right)}{\sum_{\theta} \mu(\theta) \lambda^{*}\left(\theta, p^{0}\right)}\left[1-B\left(\theta, \lambda^{*}\left(\theta, p^{0}\right), p\right)\right]=p\right\}
$$

This means that the equilibrium is not stable in the sense that starting from the neighborhood of the equilibrium state, the above update of the rational expectations on the failure probability will not converge to the equilibrium.

Our numerical results give the examples of this instability phenomenon for different initial distributions:

(i) Uniform initial distribution of $\theta$ on the interval [0,30] (Figure 1);

(ii) Gaussian initial distribution of $\theta$ on the interval $[0,30]$ with mean 15 and standard deviation 1.8 (Figure 2);

(iii) Two-sided distribution with $\mu(0)=0.3, \mu(30)=0.7$ and $\mu(\theta)=0$ for $\theta \notin\{0,30\}$ (Figure 3 ;

(iv) Gaussian initial distribution of $\theta$ on the interval $[0,200]$ with mean 100 and standard deviation 40.6 (Figure 4);

\section{Numerical Results}

In this section we discuss how the link failure probability in equilibrium changes with respect to the initial distribution. More precisely, we consider different initial distributions on some range of the capacity $\theta$. All distributions have the same mean but different variances. We choose $\theta$ in the interval $[0,30]$ and we consider a Gaussian distribution on this range with mean 15 and standard deviation $\gamma$. We vary $\gamma$ from 1 to 6 , and we find that the link failure probability in equilibrium drops from 0.14072 to 0.13748 . This shows that relatively less concentration in the initial distribution leads to less failure probability in equilibrium, see Figure 5. Moreover, the increase of the standard deviation in the initial distribution of $\theta$ leads to larger average connectivities in equilibrium. This is consistent with the results in [1] that the average connectivity is a too simple statistics of network topology, see Figure 6 . 


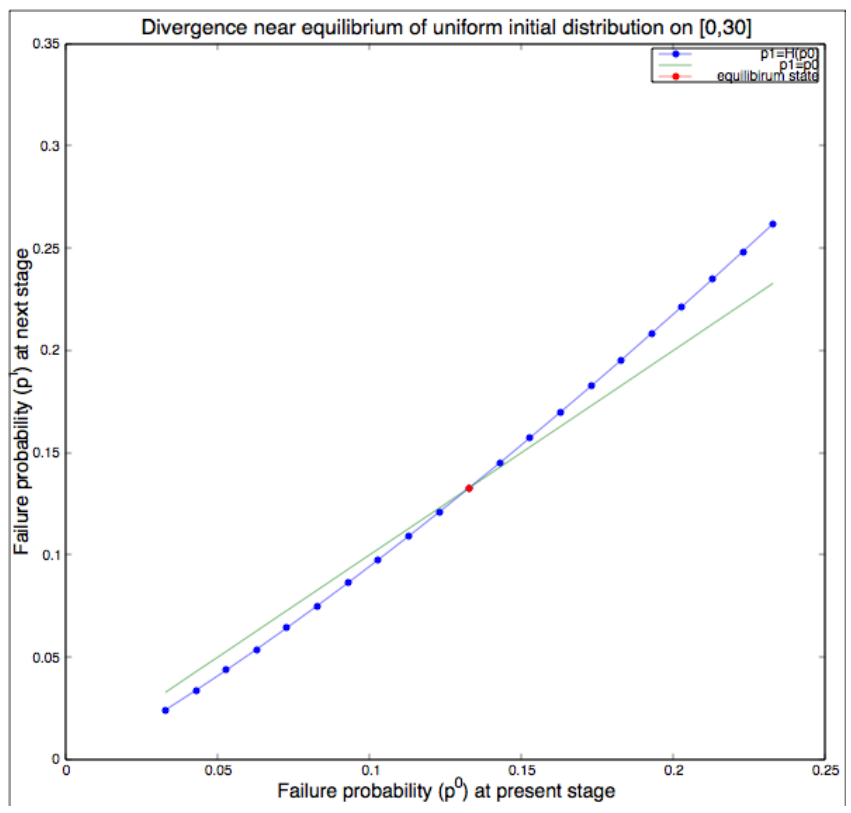

FIgURE 1. Divergence near the equilibrium state for uniform initial distribution of $\theta$ on $[0,30]$. The map $H$ has slope $>1$ near the equilibrium state.

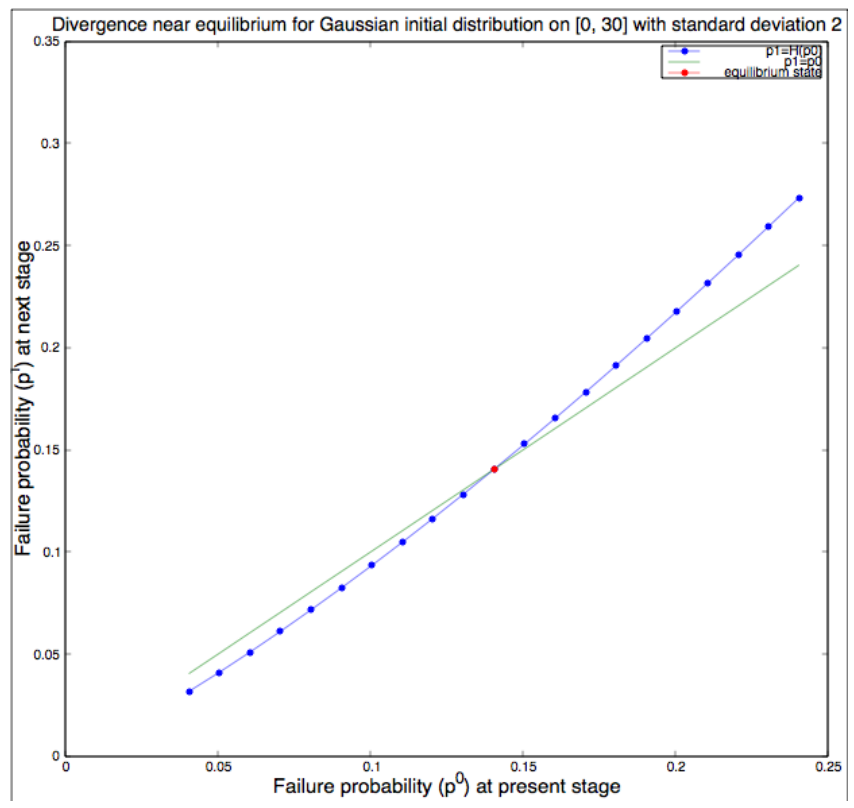

FiguRE 2. Divergence near the equilibrium state for Gaussian initial distribution of $\theta$ on $[0,30]$ (with standard deviation 2). The map $H$ has a slope $>1$ near the equilibrium state. 


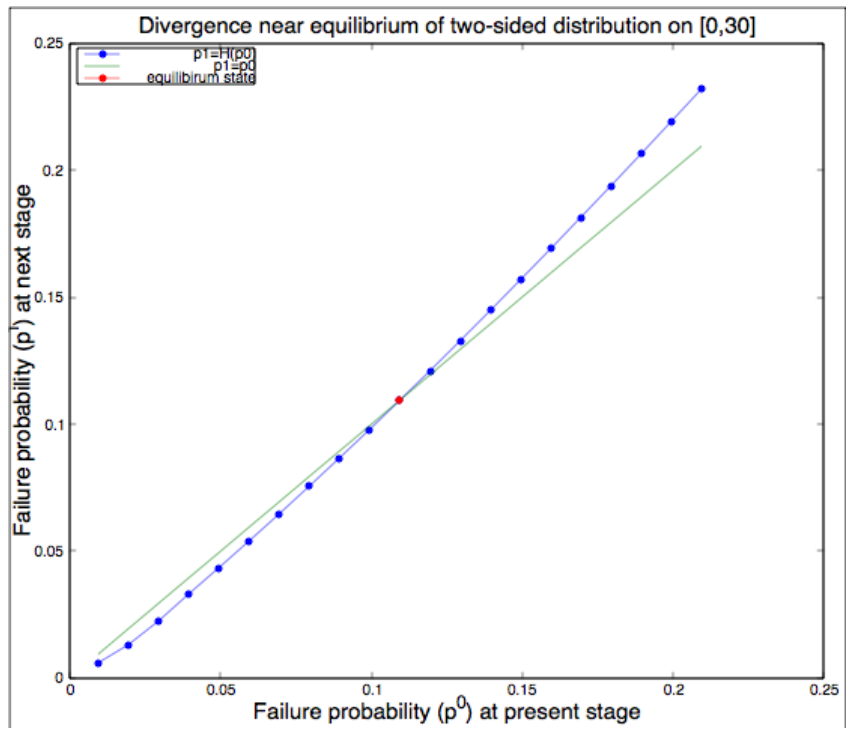

FIgURE 3. Divergence near the equilibrium state for two-sided distribution with $\mu(0)=0.3$, $\mu(30)=0.7)$. The map $H$ has a slope $>1$ near the equilibrium state.

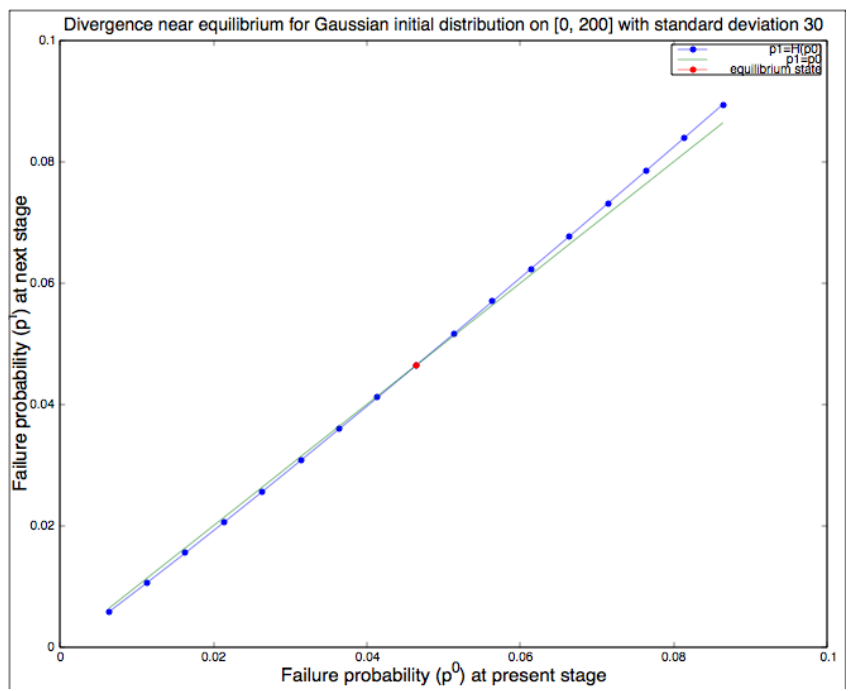

Figure 4. Divergence near the equilibrium state for Gaussian initial distribution of $\theta$ on $[0,200]$ (with standard deviation 30 ). The map $H$ has a slope $>1$ near the equilibrium state.

\section{Conclusion}

In this paper, we considered the optimal choice of connectivities by nodes in a large network. The nodes balance the benefits of connectivity with the risk of the contagion, which is endogenous and depends on the strategies of all nodes. We have studied the existence of an equilibrium in the system and the stability properties. Under full information, the equilibrium is reached simultaneously by all nodes, whereas under partial information, we show numerically that the equilibrium is unstable in the sense that a sequence of anticipated 


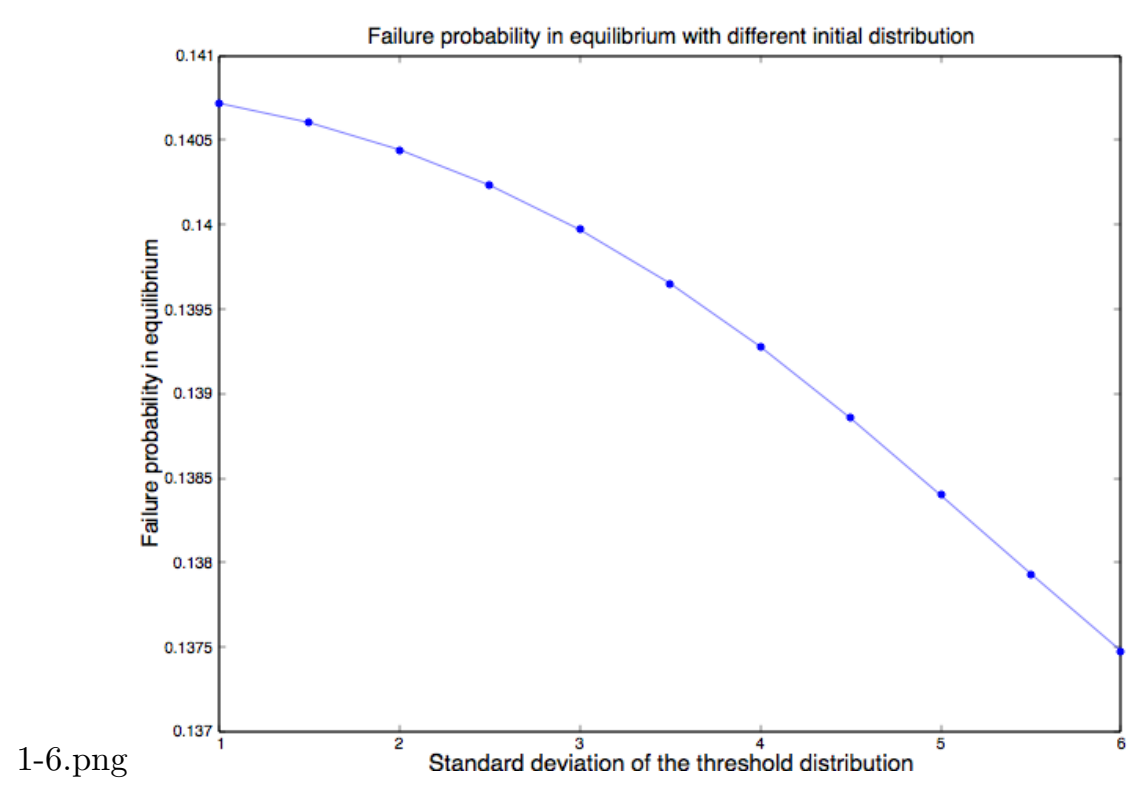

FiguRE 5. Failure probability in equilibrium with different initial distribution of $\theta$ on $[0,30]$. As the standard deviation parameter $\gamma$ increases from 1 to 6 , the failure probability in equilibrium drops from 0.14072 to 0.13748 .

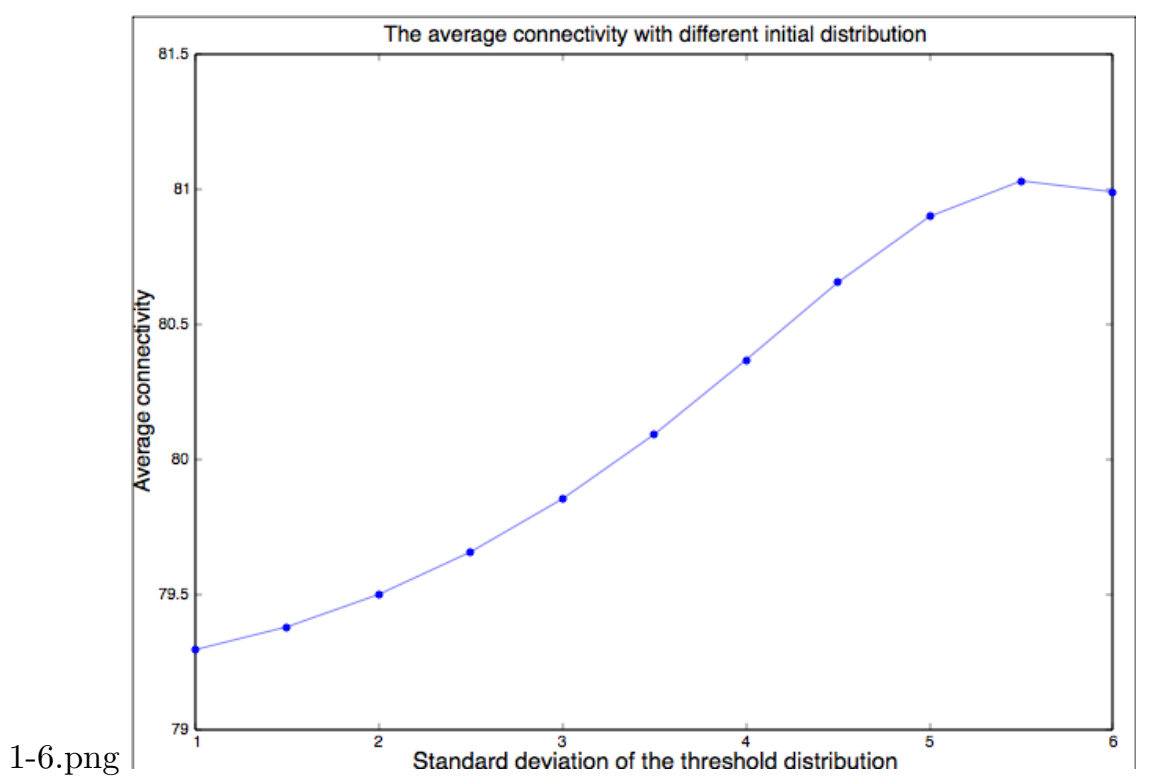

FiguRE 6 . The average connectivities of the system in the equilibrium with different initial distribution of $\theta$ on $[0,30]$. As the standard deviation parameter $\gamma$ increases from 1 to 6 , the average connectivity firstly increases from 79.295 to 81.029 then drops to 80.989 .

link failure probabilities does not converge. Our numerical results demonstrate that more concentrated distributions of threshold lead to equilibria with smaller average connectivity but higher failure probability. This 
suggests new directions of research on the relation between failure and connectivity in endogenous networks especially with dynamic feature.

\section{REFERENCES}

[1] H. Amini, R. Cont, and A. Minca. Resilience to contagion in financial networks. Mathematical Finance, 26(2):329-365, 2016.

[2] H. Amini and A. Minca. Inhomogeneous financial networks and contagious links. Operations Research, 64(5):1109-1120, 2016.

[3] H. Amini, A. Minca, and A. Sulem. Control of interbank contagion under partial information. SIAM Journal on Financial Mathematics, 6(1):1195-1219, 2015.

[4] J. Blanchet, J. Li, and Y. Shi. Stochastic risk networks: Modeling, analysis and efficient Monte Carlo, 2012. Working paper, available at https://ssrn. com/abstract $=2012987$.

[5] L. Blume, D. Easley, J. Kleinberg, R. Kleinberg, and É. Tardos. Network formation in the presence of contagious risk. In Proceedings of the 12th ACM conference on Electronic commerce, pages 1-10. ACM, 2011.

[6] R. Carmona, J.-P. Fouque, and L.-H. Sun. Mean field games and systemic risk. Communications in Mathematical Sciences, 13(4):911-933, 2015.

[7] S. Erol. Network hazard and bailouts. Working paper, 2015, rhsmith.umd.edu/files/Documents/Departments/Finance/seminars-spring2016/erol.pdf.

[8] S. Erol and R. Vohra. Network formation and systemic risk. PIER Working paper, (14-029), 2014, https://ssrn.com/abstract $=2500846$.

[9] J.-P. Fouque and T. Ichiba. Stability in a model of interbank lending. SIAM Journal on Financial Mathematics, 4(1):784-803, 2013.

[10] J. Garnier, G. Papanicolaou, and T.-W. Yang. Diversification in financial networks may increase systemic risk. Handbook on Systemic Risk, pages 432-444, 2013.

[11] J. Garnier, G. Papanicolaou, and T.-W. Yang. Large deviations for a mean field model of systemic risk. SIAM Journal on Financial Mathematics, 4(1):151-184, 2013.

[12] S. Janson. The probability that a random multigraph is simple. Combinatorics, Probability and Computing, 18(1-2):205-225, 2009.

[13] O. Kley, C. Klüppelberg, and G. Reinert. Risk in a large claims insurance market with bipartite graph structure. Operations Research, 64(5):1159-1176, 2016.

[14] A. Minca and A. Sulem. Optimal control of interbank contagion under complete information. Statistics $\&$ Risk Modeling, 31(1):23-48, 2014.

[15] L. C. Rogers and L. A. Veraart. Failure and rescue in an interbank network. Management Science, 59(4):882-898, 2013.

[16] T. Roukny, H. Bersini, H. Pirotte, G. Caldarelli, and S. Battiston. Default cascades in complex networks: Topology and systemic risk. Scientific reports, 3:2759, 2013. 